\title{
MODÉLISATION DE LA DYNAMIQUE DE L'HABITAT DES JEUNES STADES DE SAUMON ATLANTIQUE (SALMO SALAR) DE LA RIVIĖRE ASHUAPMUSHUAN (QUÉBEC, CANADA).
}

\author{
M. LECLERC (1), P. BOUDREAU (1), J. BECHARA (1), L. BELZILE (2) \\ et D. VILLENEUVE (3)
}

(1) Institut National de la Recherche Scientifique (INRS-Eau), 2800 rue Einstein, Suite 105, Québec, (Qc), Canada. G1V 4C7.

(2) Groupe Environnement Shooner Inc., 5355 boulevard des Gradins, Québec, (Qc), Canada. G2J $1 \mathrm{C} 8$.

(3) Vice-présidence Environnement, Hydro-Québec, 1010 rue Sainte-Catherine est, Montréal, (Qc), Canada. H2L 2 G3.

\section{RÉSUMÉ}

La technique de modélisation des microhabitats sert à évaluer l'impact des divers usages de l'eau sur la disponibilité de l'habitat des poissons. Cependant, un aspect négligé par ces modèles est que les variations de débit peuvent affecter aussi la position de ces habitats dans la rivière. Une approche numérique est proposée afin de simuler le déplacement des microhabitats à l'aide d'un modèle hydrodynamique bi-dimensionnel et d'une analyse hydrologique des changements temporels du débit. Cette approche a été employée lors des études d'avant-projet de l'aménagement hydroélectrique de la rivière Ashuapmushuan, dans la région du lac Saint-Jean (Québec, Canada). Dans le cadre d'une approche contrastée, le but était d'évaluer les impacts éventuels de régimes d'exploitation sur l'habitat des juvéniles du saumon atlantique (ouananiche) de cette rivière. Dans une première étape, la qualité de l'habitat d'un tronçon de rivière modélisé est évaluée pour 36 débits différents à l'aide d'indices d'habitat. Deuxièmement, un certain nombre de zones homogènes sont circonscrites à l'intérieur du tronçon modélisé, chacune portant un centroïde calculé à partir des indices d'habitat. Par la suite, le taux de mobilité de chaque centroïde est calculé en fonction du débit. Finalement, la vitesse de déplacement des centroïdes (en mètres par heure) est déterminée pour les régimes hydrologiques naturel et d'exploitation. Dans le cas hypothétique extrême du régime de pointe, le taux de déplacement de l'habitat de juvéniles serait en moyenne augmenté de 15 fois. Les résultats de la modélisation d'habitat, ainsi que d'autres évaluations d'impact environnemental, ont permis d'exclure dès le début des études le régime de pointe comme type d'exploitation hydroélectrique de la rivière Ashuapmushuan.

Mots-clés : modélisation des microhabitats, régimes de pointe, Salmo salar, rivières, Québec, hydraulique fluviale, éléments finis, habitats.

\section{A MODEL OF HABITAT DYNAMICS \\ APPLIED TO LANDLOCKED ATLANTIC SALMON (SALMO SALAR) JUVENILES OF THE ASHUAPMUSHUAN RIVER (QUEBEC, CANADA).}

\author{
ABSTRACT \\ The IFIM (Instream Flow Incremental Methodology) and its PHABSIM model \\ (Physical Habitat Simulation) have been widely used to evaluate the impact of various water \\ resource projects on the availability of fish habitat. However, these models do not consider
}


the displacement of habitat positions within the river that accompanies discharge changes. A numerical approach was developed to simulate the habitat displacement using a twodimensional hydrodynamical model combined with a hydrological analysis of temporal changes in discharge. This methodology was employed in a preliminary evaluation of the eventual impact of a peak hydropower project on the juvenile habitats of the landlocked salmon ("ouananiche") of the Ashuapmushuan River (Saint-Jean Lake region, Quebec, Canada). In a first step, the habitat quality of a modelled reach is estimated for 36 simulated discharges using the Habitat Suitability Index (HSI) approach. Second, a number of relatively homogeneous zones are defined within the modelled reach, each of them carrying a centroid calculated from the HSI. Third, the characteristic rate of mobility of each centroid as a function of discharge variations is used to calculate a velocity of habitat displacement (in metres per hour). Finally, the mobility of the centroids under natural and an extreme peak exploitation hydrological regimes are compared. Under the hypothetical peak exploitation regime, the mean rate of juvenile salmon habitat displacement of the Ashuapmushuan River would increase by a factor of 15 respective to the natural conditions. The results of the habitat modelling, as well as other environmental impact analyses, lead to the exclusion of the peak exploitation regime from the beginning of the studies.

Key-words : habitat modelling, peak exploitation, Salmo salar, rivers, Quebec.

\section{INTRODUCTION}

La modélisation des microhabitats ("Instream Flow Incremental Methodology", IFIM ; BOVEE, 1978), et son modèle PHABSIM (Physical Habitat Simulation), est une des approches les plus utilisées pour la prédiction de l'impact des changements de régime de débit sur les populations des poissons en rivière. Cette technique comporte l'élaboration d'un modèle hydrodynamique visant à prédire les vitesses et les profondeurs, ainsi que d'un modèle biologique permettant d'évaluer les conditions de l'habitat d'une espèce cible par rapport à ces variables. Les deux modèles peuvent être combinés pour déterminer une Aire Pondérée Utile (APU) qui donne une mesure de la qualité globale de l'habitat. Les variations des $A P U$ en fonction du débit aident à établir les débits souhaitables minimums et maximums pour la conservation des habitats des poissons lors de la détermination des débits réservés et d'exploitation. Cette approche a été fréquemment appliquée en Amérique du Nord (GORE, 1989 ; LECLERC et al., 1991b ; NESTLER et al., 1989 ; REISER et al., 1989) et en Nouvelle-Zélande (SCOTT et SHIRVELL, 1987) afin de déterminer les débits réservés pour la conservation de l'habitat des poissons et des organismes benthiques. L'approche a aussi été employée en Europe pour décrire l'habitat des salmonidés (SOUCHON et al., 1989).

Malgré l'utilisation répandue de cette technique, des améliorations sont souhaitables afin d'augmenter son pouvoir prédictif (MATHUR et al., 1987 ; SCOTT et SHIRVELL, 1987). Concernant le modèle hydrodynamique, LECLERC et al. (1991b) proposent une technique de modélisation bi-dimensionnelle à bancs couvrants-découvrants basée sur l'approche des éléments finis, laquelle permet de surmonter la plupart des limitations des méthodes classiques (modèles de régression, courbes de remous). De plus, la puissance des modèles bi-dimensionnels permet d'envisager d'autres applications reliées à la modélisation des microhabitats lotiques, comme il sera démontré dans cet article.

Les régimes d'exploitation dits "de pointe", caractéristiques de certaines centrales hydroélectriques, sont particulièrement difficiles à analyser avec les techniques classiques étant donné le régime particulier imposé par ces ouvrages. En effet, ce type d'exploitation comporte une production d'électricité à des heures, des jours et/ou des saisons correspondant à la plus forte demande énergétique, pouvant occasionner des variations rapides de débit et de température dans la rivière. De telles variations peuvent être nuisibles aux communautés d'invertébrés lotiques et aux populations de poissons (CUSHMAN, 1985 ; GARCÍA DE JALÓN et al., 1988 ; GISLASON, 1985 ; HVIDSTEN, 1985). Dans la modélisation des microhabitats, cette problématique a été abordée par une analyse des variations temporelles des APU selon le régime d'exploitation et la distance en aval du barrage (GORE, 1989 ; GORE et al., 1989 ; NESTLER et al., 1989). Cependant, l'évolution 
temporelle du débit dans les grandes rivières a pour effet de modifier non seulement la disponibilité et la qualité des habitats (les APU), mais également leur position dans le milieu. Ainsi, à fort débit, les meilleurs habitats à juvéniles se situent de part et d'autre du chenal d'écoulement. Par contre, à faible débit, les zones d'habitat les plus favorables ont tendance à se retrouver dans la partie centrale de l'écoulement, en raison de la réduction des vitesses et des profondeurs qui accompagne la diminution de débit. Ces déplacements s'effectuent relativement lentement, et c'est le régime hydrologique (variabilité temporelle des débits) qui en gouverne le rythme. Si ces déplacements sont anormalement rapides, ils peuvent obliger les poissons à rechercher continuellement de meilleures conditions, ou bien à subir une diminution de leur rythme de croissance due à l'occupation d'habitats devenus en moyenne moins favorables.

En conséquence, s'il est possible d'évaluer l'importance de ces déplacements et de les comparer pour des régimes naturels et d'exploitation, on pourra mieux estimer leurs répercussions environnementales. Cet article présente une approche qui permet d'obtenir une estimation du taux de déplacement de l'habitat des poissons à partir de :

- la modélisation bi-dimensionnelle des microhabitats par l'approche des éléments finis ;

- l'analyse statistique de séries hydrologiques.

Cette méthodologie a été mise à l'essai lors d'une étude d'avant-projet effectuée sur la rivière Ashuapmushuan dans la région du lac Saint-Jean (Québec, Canada), en vue d'évaluer les effets éventuels de deux centrales hydroélectriques sur l'habitat des juvéniles du saumon atlantique (Salmo salar L.) dulcicole, localement appelé "ouananiche".

\section{MATÉRIEL ET MÉTHODES}

\subsection{Site à l'étude}

La rivière Ashuapmushuan est un des plus importants affluents du lac Saint-Jean (figure 1). La géologie et la végétation de son bassin versant sont typiques du bouclier canadien, constitué d'une base de roche granitique. La forêt boréale domine au nord du bassin, avec une transition vers la forêt mixte près de l'embouchure. Le débit moyen annuel de la rivière est de $300 \mathrm{~m}^{3} / \mathrm{s}$ avec de fortes crues lors du dégel printanier (voisinant en moyenne $1000 \mathrm{~m}^{3} / \mathrm{s}$ et culminant à $2500 \mathrm{~m}^{3} / \mathrm{s}$ ) et de faibles débits à la fin de l'hiver (en moyenne, de l'ordre de $80 \mathrm{~m}^{3} / \mathrm{s}$ ).

La compagnie d'électricité Hydro-Québec a entrepris en 1989 des études d'avantprojet pour l'aménagement hydroélectrique de la rivière Ashuapmushuan. Le projet incluait la construction de deux centrales hydroélectriques (figure 1).

Aux fins de la modélisation, un secteur de la rivière fut choisi à partir de l'examen de photos aériennes et d'un survol héliporté. L'examen de photos aériennes a consisté à reconnaître les habitats propices au développement des saumons juvéniles à l'aide du système de classification hydromorphologique de COTÉ et al. (1987), qui comprend trois catégories d'aires d'élevage. Ces catégories sont établies selon le faciès d'écoulement des segments de rivière (bassin, chenal, seuil ou rapide) et la granulométrie du substrat. Ainsi, on reconnaît :

a) les aires très favorables à la croissance (qui représentent $10 \%$ de la superficie de l'Ashuapmushuan): rapides coulant sur un lit de galets ou de blocs (voir les définitions des catégories de substrats au tableau I) ;

b) les aires favorables ( $6 \%$ de la superficie) : seuils ou chenaux où dominent les graviers et les cailloux ;

c) les aires peu favorables ( $84 \%$ de la superficie) : chutes, cascades, zones fluviolacustres. 


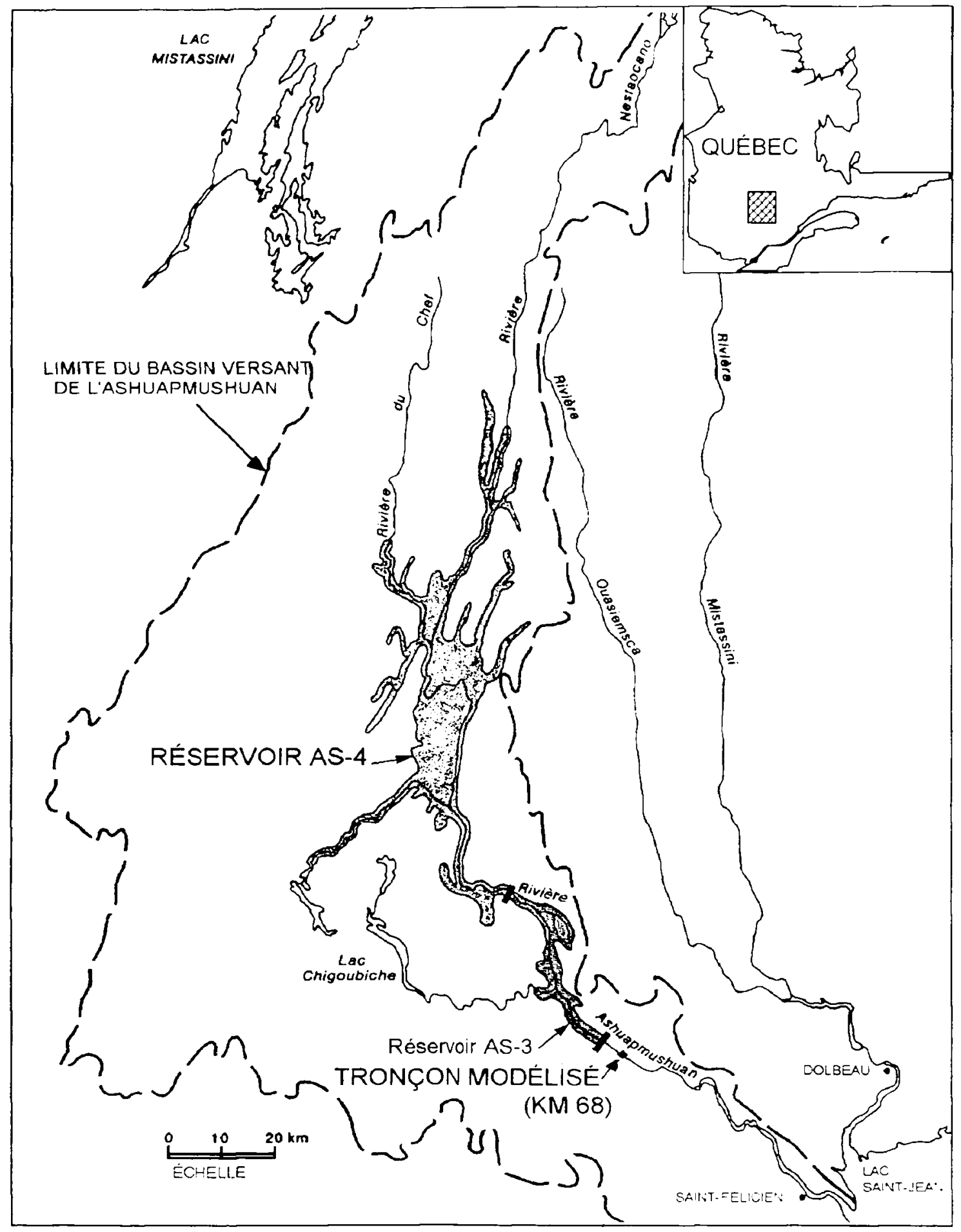

Figure 1 : Localisation du tronçon modélisé et contour approximatif des réservoirs projetés dans le bassin hydrographique de la rivière Ashuapmushuan.

Figure 1 : Geographical location of the modelled site and approximate limits of the projected reservoirs within the Ashuapmushuan River basin. 
Pour les fins de la modélisation, un site d'étude situé à $68 \mathrm{~km}$ de l'embouchure de la rivière a été choisi (figure 1). Ce site est représentatif des conditions les plus favorables pour la croissance des jeunes saumons (types a et b). II s'agit d'un segment de rivière de $1,4 \mathrm{~km}$ de longueur, de $0,245 \mathrm{~km}$ de largueur moyenne et de $0,342 \mathrm{~km}^{2}$ de superficie (à la limite du lit majeur). Sa superficie représente environ $5 \%$ du total des sites favorables et très favorables à la croissance dans la section de la rivière accessible à la ouananiche.

\subsection{Modèle hydrodynamique}

L'objectif principal de la modélisation hydrodynamique est de prédire localement la profondeur, la vitesse d'écoulement moyenne sur la verticale et la surface mouillée pour des événements (débits) dont on ne possède pas de mesures de terrain. Les relations mathématiques décrivant les écoulements sont celles de Saint-Venant (LECLERC et al., 1987,1990 ). Ces équations respectent les principes de conservation de la masse (débit) et de la quantité de mouvement et sont résolues avec la méthode des éléments finis (DHATT et TOUZOT, 1981). La capacité de déterminer par lui-même la position du littoral du cours d'eau en fonction du débit (modèle à bancs couvrants-découvrants) constitue l'essentiel de l'originalité du modèle employé. Cette caractéristique est particulièrement utile sur des milieux à frontière mobile (estuaires à marée, rivières, fleuves) dont le régime hydrologique peut faire varier significativement l'aire mouillée par l'écoulement. Pour une description plus complète du modèle, le lecteur peut consulter LECLERC et al. $(1987,1990)$.

L'application du modèle hydrodynamique comprend quatre étapes fondamentales : 1) les relevés sur le terrain ; 2) le modèle numérique de terrain ; 3) la calibration ; 4) la validation.

\subsubsection{Relevés sur le terrain}

Deux ensembles de données physiques sont nécessaires pour construire le modèle numérique de terrain : 1) une carte bathymétrique et une carte des substrats du site à modéliser ; 2) quelques mesures (minimum deux événements distincts) du niveau et du débit correspondant aux frontières amont et aval du site. Pour dresser les cartes du site choisi (figure 2A,B), le niveau géodésique du fond a été mesuré et le substrat a été caractérisé en 2600 points d'échantillonnage (environ une mesure par $100 \mathrm{~m}^{2}$ ) par identification visuelle des classes dominantes (maximum cinq), auxquelles on a associé une valeur médiane de taille en $\mathrm{mm}$ (tableau I). Pour obtenir le diamètre moyen d'un

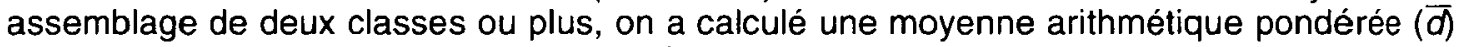
qui tient compte du nombre de classes présentes et de leur ordre d'importance $i$ :

$$
\bar{d}=\sum_{i=1}^{p} w_{i} d_{i}
$$

où $d_{i}$ est la valeur médiane de la classe i sur l'échelle donnée au tableau I;

$p \quad$ est le nombre de classes identifiées en un lieu particulier ;

$w_{i}$ est la valeur des pondérations utilisées selon le nombre de classes (selon

LECLERC et al., 1991a).

Deux données de jaugeages (ceux correspondant à la calibration et à la validation du modèle) ont servi à établir la dénivellation de la ligne d'eau du site. La dénivellation étant semblable lors des deux jaugeages, la même valeur a été utilisée pour chacune des simulations effectuées. Cependant, pour que les résultats de la modélisation soient valables, la dénivellation totale observée sur le terrain entre les deux extrémités du site doit demeurer constante pour la gamme de débit simulée (écoulement quasi-uniforme). Dans le cas du site d'étude, cette supposition est apparue acceptable à la suite d'une observation détaillée du lit dans les régions frontières amont et aval du site. De plus, le modèle numérique devient souvent instable lorsque les conditions imposées ne conduisent pas à une solution acceptable du problème posé. 
Tableau I : Définition de catégories granulométriques employées (source : LECLERC et al., 1991a). Le diamètre médian est calculé selon l'échelle de phi (phi est égale au logarithme négatif en base 2 de la taille de la particule en millimètres).

Table I : Granulometric categories employed (source : LECLERC et al., 1991a). Median diameter is calculated following the phi scale (where phi is equal to the negative logarithm in base 2 of the particle size in millimetres).

\begin{tabular}{|c|c|c|c|}
\hline Type de sédiment & Abréviation & $\begin{array}{l}\text { Diamètre } \\
(\mathrm{mm})\end{array}$ & $\begin{array}{l}\text { Diamètre médian } \\
(\mathrm{mm})\end{array}$ \\
\hline Blocs métriques & $\mathrm{B}^{+}$ & $>1000$ & 1000 \\
\hline Blocs & $B$ & 250 à 1000 & 500 \\
\hline Galets & $\mathrm{G}$ & 64 à 250 & 125 \\
\hline Cailloux & C & 32 à 64 & 45 \\
\hline Gravier & V & 4 à & 10 \\
\hline Sable & $\mathrm{S}$ & 0,062 à & 0,5 \\
\hline
\end{tabular}

Afin de calibrer et valider le modèle, un total de 46 mesures de vitesse moyenne et de niveau d'eau au site ont été effectuées à un débit de $184 \mathrm{~m}^{3 / \mathrm{s}}$ (21 juillet 1989) pour la calibration et à un débit de $146 \mathrm{~m}^{3} / \mathrm{s}$ (26 juillet 1989) pour la validation. La vitesse a été mesurée en deux points de la colonne d'eau, soit à $0,8 H$ et $0,2 H$ ( $H$ étant la profondeur de la colonne d'eau), avec un courantomètre de marque $A$. Ott Z-100, avant d'être transformée en valeur moyenne.

\subsubsection{Modèle numérique de terrain}

Le modèle numérique de terrain constitue le support informatique du modèle hydrodynamique. Il est le résultat de la "discrétisation" (découpage) du domaine d'écoulement en un certain nombre d'éléments géométriques de forme triangulaire et portant chacun six noeuds, trois situés aux sommets et trois aux milieux des côtés (figure 2A). La vitesse du courant est calculée aux six noeuds, la hauteur d'eau et la profondeur sont évaluées aux trois noeuds-sommets et le substrat est spécifié comme une valeur constante sur chaque élément. A l'intérieur de chaque élément, les variables sont interpolées en fonction de la position relative du point considéré permettant d'obtenir les vitesses pour l'ensemble du domaine (figure $2 \mathrm{C}$ ). Le site à l'étude a été discrétisé en 1659 éléments et 3444 noeuds (figure 2A).

\subsubsection{Calibration et validation}

La calibration consiste essentiellement à ajuster les paramètres de frottement (" $n$ " de Manning) et de viscosité turbulente (coefficient numérique de viscosité turbulente) pour un événement hydrologique de référence (LECLERC et al., 1987, 1990). A cette fin, les valeurs mesurées et calculées des vitesses et des niveaux d'eau sont comparées. Les coefficients sont ajustés selon le diamètre moyen obtenu par l'équation [1] jusqu'à obtenir des résultats stables et consistants lors des simulations numériques. Le tableau II donne la valeur finale des coefficients de Manning retenus pour les différents assemblages granulométriques. Pour la viscosité numérique turbulente, on a retenu une valeur constante de $1 \mathrm{~m}^{2} / \mathrm{s}$.

La validation consiste à comparer les données mesurées et calculées pour un ou des événements de référence indépendants de celui de la calibration. Des erreurs moyennes de $4,7 \mathrm{~cm}$ pour les niveaux d'eau et de $0,10 \mathrm{~m} / \mathrm{s}(10,4 \%)$ pour les vitesses ont été obtenues. L'ordre de grandeur de ces erreurs est typique de la méthode employée (LECLERC et al., 1991b). 

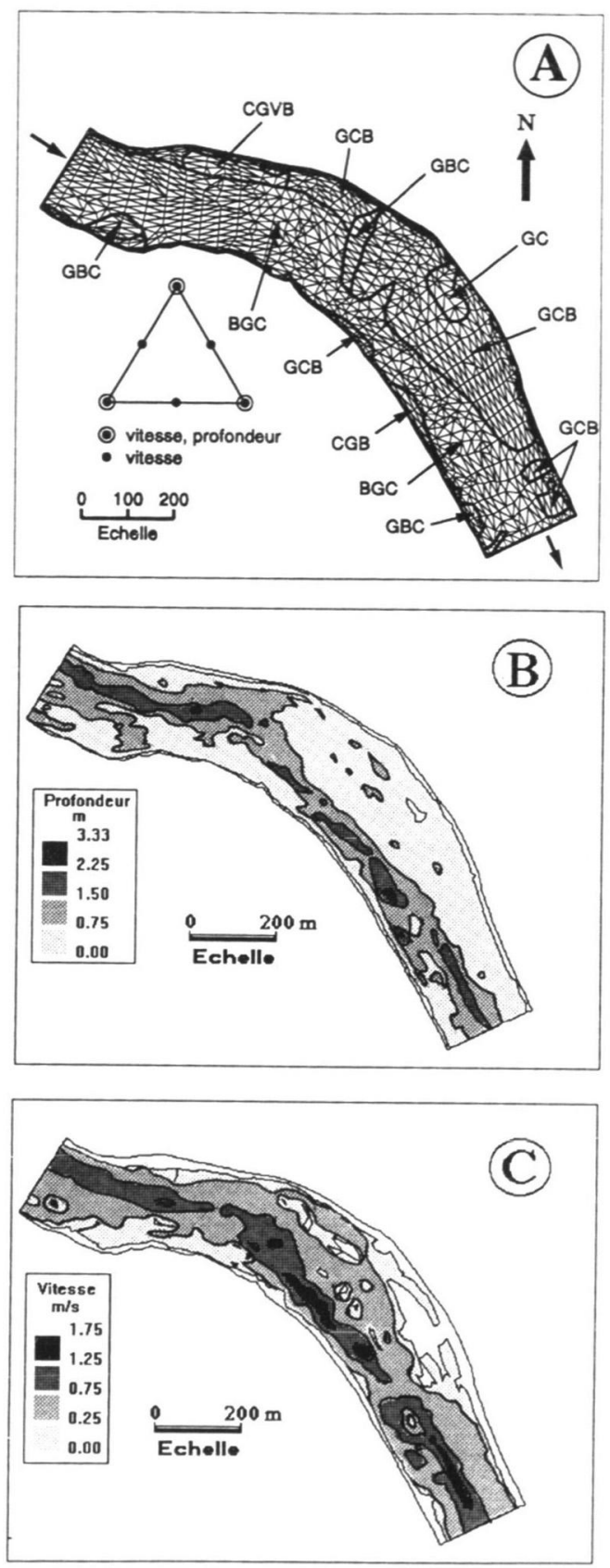

Figure 2 : Divers aspects du modèle hydrodynamique du site. A) Domaine d'écoulement discrétisé et principaux assemblages granulométriques. Le triangle représente un élément (fini) aux nœuds duquel sont prédites la vitesse et la profondeur. Les flèches aux extrémités du domaine indiquent la direction de l'écoulement ; B) Carte bathymétrique (profondeur en mètres pour un débit de $192 \mathrm{~m}^{3} / \mathrm{s}$ ) ; C) Résultat d'une simulation des vitesses et des frontières de l'écoulement pour un débit de $73 \mathrm{~m}^{3} / \mathrm{s}$.

Figure 2 : Different aspects of the hydrodynamic model of the site. A) Discretized flow domain and substrate. The triangle represents an element with the nodes to calculate velocity and depth. Small arrows indicate flow direction ; B) Bathymetry (depth in metres corresponding to a discharge of $192 \mathrm{~m}^{3} / \mathrm{s}$ ) ; C) Simulated velocities and flow boundaries for a discharge of $73 \mathrm{~m}^{3} / \mathrm{s}$. 
Tableau II : Coefficients de Manning ( $n$ ) retenus selon le type de substrat après la calibration et indices de base de substrat pour les assemblages granulométriques du site à l'étude. $\bar{d}(\mathrm{~cm})$ : diamètre moyen du substrat. Les lettres majuscules correspondent aux catégories de substrats représentées au tableau $I$.

Table II : Intervals of the Manning's coefficients $(n)$ retained after calibration, and HSI corresponding to each of the granulometric assemblages found in the study site. $\bar{d}(\mathrm{~cm})$ : mean substrate diameter. See table I for an explanation of the capital letters corresponding to substrate categories.

\begin{tabular}{ccccc}
\hline $\begin{array}{c}\text { Type de } \\
\text { substrat }\end{array}$ & $\bar{d}(\mathrm{~cm})$ & $n$ de Manning & \multicolumn{2}{c}{ Indices de base du substrat } \\
\cline { 4 - 5 } BG & 35.0 & 0,038 & 0.07 & 0.50 \\
BGC & 29.7 & $0.038-0.034$ & 0.10 & 0.50 \\
GBC & 22.2 & 0,035 & 0.19 & 0.38 \\
GCB & 17.6 & 0,033 & 0.38 & 0.75 \\
GC & 9.3 & 0,029 & 0.50 & 0.75 \\
GCV & 7.8 & 0,028 & 0.75 & 0.75 \\
GCVB & 11.6 & 0,030 & 0.75 & 0.75 \\
CGB & 16.0 & 0,032 & 0.50 & 1 \\
CGVB & 10.8 & 0,029 & 1 & 1 \\
CVG & 5.1 & 0,026 & 1 & 1 \\
VGB & 14.3 & 0,031 & 0.50 & 0.75 \\
SGB & 13.9 & 0,031 & 0 & 0.25 \\
SGC & 4.8 & 0,026 & 0 & 0.25 \\
SCG & 4.0 & 0,025 & 0 & 0.25 \\
SVCG & 2.6 & 0,023 & 0 & 0.25 \\
Roc & - & 0,045 & 0 & 0 \\
\hline
\end{tabular}

\subsection{Modèle ichtyologique}

\subsubsection{Choix d'une espèce cible}

La "ouananiche" a été choisie comme espèce cible pour l'analyse des habitats, étant donné sa grande importance socio-économique pour la région du lac Saint-Jean. Après leur naissance, les juvéniles (stades alevin et tacon) restent dans la rivière Ashuapmushuan et ses affluents pendant une durée moyenne de 2 à 3 ans, et dévalent par la suite vers le lac Saint-Jean pour poursuivre leur croissance. Les ouananiches séjournent dans le lac pendant une période de 2 à 3 ans en général, puis, devenues matures sexuellement, reviennent à la rivière pour se reproduire. Dans le contexte de la présente étude, les phases du cycle vital les plus sensibles aux ouvrages hydroélectriques projetés correspondent à la période de croissance des juvéniles et à la reproduction. La modélisation des frayères est décrite dans LECLERC et al. (1991a).

\subsubsection{Relevés de terrain et élaboration des indices de sélectivité}

Nous avons employé une version modifiée de l'approche des "indices de sélectivité d'habitat" (ISH), connus comme "Habitat Suitability Index" (HSI) dans la littérature anglophone (BOVEE, 1978). A chaque phase sensible du cycle vital d'une espèce, correspondent un groupe spécifique d'indices de base $\left(I_{S}, I_{H}, I_{V}\right)$ et un indice global $\left(I_{G}\right)$, résultant de la combinaison algébrique de plusieurs indices de base. Ces derniers sont élaborés pour des variables de l'environnement reliées au débit (profondeur, vitesse moyenne sur la verticale et substrat), à partir des observations de poissons dans leur milieu naturel. La fréquence d'utilisation des différents habitats est ensuite pondérée par la 
disponibilité relative de ceux-ci dans le milieu échantillonné. L'indice final varie entre 1 (excellent habitat) et 0 (mauvais habitat) selon que les conditions sont celles où les poissons ont été les plus fréquemment observés, ou celles où aucun poisson n'a été observé, respectivement. Les différents indices de base sont par la suite combinés dans une moyenne géométrique pondérée (indice global). Ces pondérations sont obtenues en affectant à chacun des indices de base un exposant obtenu d'après une analyse en composantes principales qui permet d'estimer la contribution relative de chaque variable physique à la variabilité des sites d'échantillonnage.

Afin d'élaborer les courbes de sélectivité, on a effectué des relevés de terrain entre le 17 et le 30 juillet 1989 sur deux sites d'échantillonnage situés sur le cours principal de l'Ashuapmushuan ( $\mathrm{km} 50$ et 68). Malgré un effort d'échantillonnage comprenant 335 parcelles d'observation, seulement 26 poissons ont été trouvés sur les deux sites ; ce qui est insuffisant pour élaborer des indices de sélectivité. Cette faible abondance est probablement liée à une diminution des stocks de ouananiche au lac Saint-Jean (VALENTINE, 1991) et non pas à la qualité de l'habitat échantillonné. Comme alternative, il a été décidé de recourir en grande partie au modèle d'habitat du saumon atlantique anadrome établi par BOUDREAULT et al. (1989) sur la rivière Moisie (Québec, Canada). Ce choix, applicable à la vitesse et à la profondeur, se justifie d'abord parce que ce modèle s'applique à la même espèce, et que la taille et l'aspect de la Moisie sont similaires à ceux de l'Ashuapmushuan. De plus, des tests de comparaison de la distribution de fréquences des vitesses et des profondeurs mesurées dans les deux rivières ont démontré qu'il n'y avait pas de différences significatives (Test de Kolmogorov-Smirnov, approximation de $X^{2}=1,73$ et $0,29, P>0,05$, vitesse et profondeur respectivement). Ces indices ont été développés pour les alevins $(0+a n, 3-5 \mathrm{~cm}$ de longueur), et les tacons ( $1+$ et $2+$ ans, plus de $6 \mathrm{~cm}$ ).

Toutefois, en ce qui concerne les substrats, une différence significative a été décelée (Test de Kolmogorov-Smirnov, approximation de $X^{2}=277,95, P<0,01$ ) et la préférence des juvéniles pour cette variable a plutôt été quantifiée à l'aide d'un système de classification visant à déterminer individuellement la valeur salmonicole des différents assemblages granulométriques. Les résultats de cette classification sont montrés au tableau II. Le système de classification a été élaboré d'une part à partir des observations faites lors des relevés de terrain, et d'autre part, à partir des informations contenues dans la bibliographie à ce sujet (entre autres: HAVEY et WARNER, 1970 ; SYMONS et HELAND, 1978 ; RIMMER, 1980). L'indice de base de substrat $I_{S}$ est évalué en tenant compte du rang $i$ d'importance de la classe de granulat dans l'assemblage, de la valeur individuelle $s_{i}$ des classes présentes et d'un indice de diversité $I_{D}$. II s'obtient de la façon suivante:

$$
I_{S}=I_{0} \times s_{1} \times s_{2} \times s_{3}
$$

L'indice de diversité $I_{D}$ est égal à 1 lorsque trois classes de substrat ou plus sont présentes et il prend les valeurs respectives de 0,66 et 0,33 lorsque seulement deux ou une classes sont observées en un lieu, respectivement. Cette cotation repose sur le postulat que plus l'assemblage granulométrique est diversifié, plus il devrait être favorable pour l'alimentation et le développement des juvéniles. En effet, un substrat hétérogène supporterait plus d'insectes aquatiques, source de nourriture pour les juvéniles, qu'un substrat homogène (MINSHALL, 1985). De plus, il est permis de présumer qu'un substrat varié offrira également plus de possibilités d'abris et de microhabitats aux juvéniles qu'un lit composé uniquement d'un seul type de sédiment. Les valeurs des sous-indices $s_{i}$ sont données dans LECLERC et al. (1991a).

Les exposants utilisés pour la moyenne géométrique pondérée (indice global, $I_{G}$ ) sont ceux qui avaient été établis pour l'étude du saumon de la rivière Moisie (BOUDREAULT et al., 1989). Les indices globaux $\left(I_{G}\right)$ des différentes phases du cycle vital de la ouananiche sont donnés par les formules suivantes:

$$
\begin{aligned}
& \text { Alevins : } I_{G A}=I_{V}^{0,44} \times I_{H}^{0,26} \times I_{S}^{0,30} \\
& \text { Tacons: } I_{G T}=I_{V}^{0,39} \times I_{H}^{0,40} \times I_{S}^{0,21}
\end{aligned}
$$

où $I_{V}, I_{H}, I_{S}$ sont les indices de base respectivement pour la vitesse, la profondeur et le substrat. 


\subsubsection{Calcul des aires d'habitat sur un site}

La méthode d'interpolation par éléments finis permet d'obtenir algébriquement la distribution spatiale de la valeur de chaque variable ou des indices de base qui en découlent. Pour le calcul de la valeur de la disponibilité de l'habitat correspondant à un débit donné, on a déterminé les valeurs suivantes:

- aire pondérée utile $(A P U)$, qui représente la superficie du cours d'eau pondérée par la valeur locale de l'habitat d'après un indice global $\left(I_{G}\right)$; cette aire est exprimée en termes absolu ou relatif (pourcentage de la superficie totale du milieu modélisé ou $A P U \%$ ) ;

- aires correspondant à des intervalles de valeurs d'habitat données $\left(I_{G}\right)$.

Pour calculer ces quantités, on utilise une méthode d'intégration numérique faisant appel à un échantillon précis de points sur un élément (12 points dans le cas présent) et à une pondération appropriée définissant le degré de représentativité du point d'intégration sur l'élément (méthode de Hammer; DHATT et TOUZOT, 1981). Par exemple, pour calculer l'aire pondérée utile (APU) d'un site, on applique la formule suivante :

$$
A P U=\sum_{i=1}^{N E} A P U_{i}=\sum_{i=1}^{N E} A_{i} \sum_{j=1}^{N P G} w_{j} l_{G i j}
$$

où $A_{i} \quad$ est l'aire d'un élément ;

$I_{G}$ est la valeur de l'indice global ;

$N E$ est le nombre total d'éléments du maillage ;

$N P G$ est le nombre de points d'intégration sur un élément $(=12)$;

wj sont les poids d'intégration.

Pour classifier les aires d'un site selon la valeur d'un indice, on procède de façon similaire en cumulant par intervalles de classe les valeurs d'aires individuelles des points d'intégration (données par leur poids et l'aire de l'élément où ils se trouvent).

\subsection{Dynamique de l'habitat}

\subsubsection{Taux de déplacement des habitats en fonction du débit}

L'influence de l'évolution du débit sur la disponibilité et la position de l'habitat est illustrée à la figure 3. Le modèle développé permet de décrire la vitesse à laquelle le centre (centroïde) d'une zone d'habitat se déplace avec le débit.

\subsubsection{Zones homogènes}

Pour calculer le taux de déplacement des habitats en fonction du régime hydrologique, on cherche d'abord à connaître la position moyenne de l'habitat en fonction du débit à l'intérieur d'une zone homogène. Comme les déplacements d'habitat se font principalement selon un axe transversal à l'écoulement, et vers le centre ou la berge selon que le débit diminue ou augmente, l'axe central de l'écoulement (le thalweg) constitue de façon évidente une limite longitudinale de zone (figure 3). Au sein des deux régions ainsi définies, on doit encore subdiviser le milieu selon des limites plus ou moins transversales pour tenir compte de son hétérogénéité dans le sens longitudinal. Enfin, la taille des zones doit être d'une grandeur suffisante pour englober assez largement le rayon de déplacement naturel des juvéniles en période d'alimentation dans un intervalle de temps court (l'heure ou la journée). Six zones distinctes pour la croissance des juvéniles ont ainsi été définies (figure 6).

\subsubsection{Méthode de calcul}

Le taux de déplacement de l'habitat dans une zone homogène a été estimé à partir de la position moyenne de l'habitat, appelée ci-après le centroïde ; celui-ci étant lié au débit, il se 

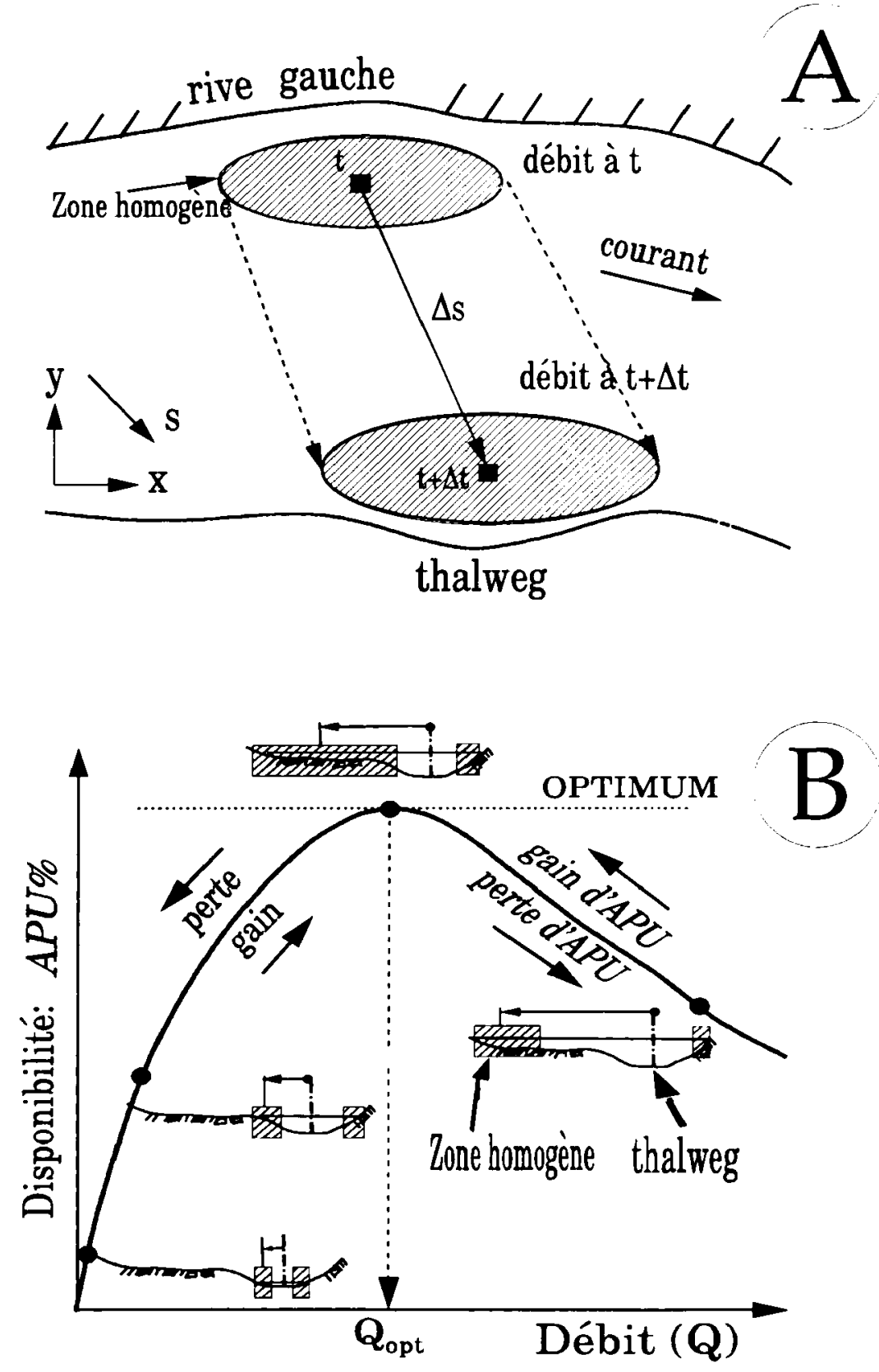

Figure 3 : Représentation simplifiée de l'influence du régime hydrologique sur la disponibilité et les déplacements d'habitat. A) Déplacement d'une zone homogène en fonction du débit ; B) Variations de l'aire et de la position occupée par les zones homogènes en fonction des APU. Le thalweg représente l'axe central de l'écoulement. Voir la section 2.4. Dynamique de l'habitat, pour une description des termes et symboles employés.

Figure 3 : Simplified scheme representing the influence of the hydrological regime on the availability and the displacement of the habitat. A) Displacement of an homogeneous zone with discharge ; B) Variations in area and positions of homogeneous zones with changes in PUA (Percent Usable Area). See section 2.4. Dynamique de l'habitat, for a description of the symbols and terms employed. 
déplacera en fonction du régime hydrologique de la rivière. Pour un débit donné, cette position est obtenue en appliquant la fonction d'intégration suivante sur l'ensemble d'une zone :

$$
\overline{(x, \bar{y})}=\frac{\sum_{i=1}^{N Z} \sum_{j=1}^{N P G} A P U_{i j}(x, y)_{i j}}{A P U}
$$

où $A P U_{i j}$ est la valeur en aire pondérée utile d'un point d'intégration ;

$i \quad$ est l'indice de l'élément (unité de surface);

j est l'indice du point d'intégration sur un élément (point de calcul, 12 par élément) ;

$N P G$ est le nombre de points d'intégration sur un élément $(=12)$;

$N Z$ est le nombre d'éléments de la zone considérée ;

$(\bar{x}, \bar{y})$ sont les coordonnées moyennes des habitats de la zone ;

$(x, y)_{i j}$ sont les coordonnées du point d'intégration sur un élément.

La position moyenne ainsi obtenue est spécifique au débit considéré, lequel varie dans le temps (figure 3A). Dans la mesure où une variation de débit dans une zone entraîne un déplacement des habitats et que ce déplacement s'effectue dans un intervalle de temps donné, il en résulte une vitesse de déplacement des habitats qui peut être calculée ainsi :

$$
\begin{aligned}
& D=\frac{\Delta \bar{s}}{\Delta t}=M \frac{\Delta Q}{\Delta t}=M \delta Q \\
& \text { avec } M=\operatorname{moy} \frac{\Delta \bar{s}}{\Delta Q}
\end{aligned}
$$

où $D$ est la vitesse de déplacement des habitats ( $\mathrm{m} / \mathrm{h}$ ou $\mathrm{m} / \mathrm{jour}$ ) ;

$M$ est le taux caractéristique de mobilité de l'habitat dans une zone en fonction du débit $\left(\mathrm{m} / \mathrm{m}^{3} / \mathrm{s}\right)$;

$\bar{S}$ est une coordonnée curviligne locale dans le sens du déplacement de l'habitat ;

$\triangle Q$ est une variation du débit $\left(\mathrm{m}^{3} / \mathrm{s}\right)$;

$\Delta t$ est un intervalle de temps (jour, heure);

$\delta Q$ est le taux de variation du débit $\left(\mathrm{m}^{3} / \mathrm{s} / \mathrm{h}\right.$ ou $\left.\mathrm{m}^{3} / \mathrm{s} / \mathrm{jour}\right)$.

Le taux de mobilité $M$ est obtenu par la méthode d'analyse spatiale des données de simulation exposée précédemment et peut être considéré comme un paramètre constant pour une zone donnée; ce qui se justifie tant que la morphologie du lit ne change pas de façon très marquée avec le débit. Dans la présente étude, seuls les meilleurs habitats $\left(I_{G}>0,7\right)$ ont été considérés dans les calculs.

\subsubsection{Taux de variation du débit}

Le taux de variation $\delta Q$ du débit dépend des séries temporelles utilisées (naturelles, régimes d'exploitation). La méthode proposée consiste d'abord à définir la période cible (été) et par conséquent le segment de la série disponible qui sera analysé. Par la suite, on calcule la différence (en valeur absolue) de débit entre toutes les valeurs adjacentes possibles de la série. Cette série intermédiaire est ensuite divisée par l'intervalle de temps $\Delta t$ de la série (e.g., la journée, l'heure) pour donner un taux. De ce premier traitement est donc obtenue une nouvelle série constituée des taux de variation du débit représentés par la variable $\delta Q$ et de laquelle on peut tirer certaines statistiques descriptives de base (BOBÉE et ROBITAILLE, 1977). Par exemple, le taux de variation moyen journalier ou horaire $(\overline{\delta Q})$, ou les taux de variation dépassés $50 \%, 10 \%$ et $2 \%$ du temps $\left(Q_{50}, Q_{10}, Q_{2}\right)$. En se rapportant à la formule [7], on peut ainsi obtenir des vitesses de déplacement correspondant aux diverses statistiques de taux de variation du débit. 
Concernant le site à l'étude, les taux de variation du débit ont été calculés pour le régime naturel et une hypothèse de régime d'exploitation pendant la période cible de croissance des juvéniles (du 10 juin au 30 septembre). L'analyse statistique des écarts journaliers de débit en conditions naturelles a été menée sur la série temporelle de débits journaliers d'une station interpolée au kilomètre 68 de la rivière Ashuapmushuan. L'interpolation a été faite à l'aide d'une analyse de régression (GORDON et al., 1992), à partir d'une station hydrologique située sur la rivière Ashuapmushuan (Station QA061901, Gouvernement du Québec), et tient compte de la proportion du bassin versant qui draine la rivière au site à l'étude. La période d'analyse s'étend sur 27 ans (1963-1989), une durée utilisée fréquemment dans les analyses de séries hydrologiques (GORDON et al., 1992). Les statistiques des écarts journaliers sont ramenées en taux de variation horaire afin de pouvoir les comparer avec les données de l'hypothèse de régime d'exploitation.

Pour le régime d'exploitation, les taux de variation de débit ont été calculés à partir d'une adaptation de données horaires provenant de la rivière aux Outardes (Québec, Canada), laquelle est soumise à un régime d'exploitation de pointe. L'analyse statistique a été effectuée sur une série journalière reconstruite à partir des données de 1988 du barrage Outardes IV. Pour un jour typique du mois d'août par exemple, le débit tôt le matin peut passer de 48 à $197 \mathrm{~m}^{3} / \mathrm{s}$ dans l'intervalle d'une heure, diminuant à $149 \mathrm{~m}^{3} / \mathrm{s}$ le soir, et augmentant à nouveau à $220 \mathrm{~m}^{3} / \mathrm{s}$ pendant une partie de la nuit (LECLERC et al., 1991a).

\section{RÉSULTATS}

\subsection{Influence du débit sur l'habitat}

Disposant d'un modèle représentatif de la courantométrie du site (figure 2C), il est possible de procéder à la simulation de conditions correspondant à des débits n'ayant pas fait l'objet de mesures détaillées. En s'inspirant des études hydrologiques et compte tenu des limites d'application du modèle hydrodynamique (LECLERC et al., 1987, 1990), des simulations des courants ont été effectuées pour une gamme de débits s'échelonnant entre 73 et $508 \mathrm{~m}^{3} / \mathrm{s}$ (36 valeurs distinctes). Cette gamme est représentative des épisodes hydrologiques de la période estivale à la hauteur du $\mathrm{km} 68$ de la rivière. Cette gamme peut également représenter la majorité des événements correspondant à une hypothèse de régime de pointe (en particulier, les étiages). Ces événements ont ensuite fait l'objet de simulations des conditions d'habitat (seulement dix de ceux-ci sont représentés sur les courbes à venir de la figure 5).

\subsubsection{Illustration de l'influence du débit sur l'habitat}

Avant l'analyse quantitative des résultats, il convient de présenter les données obtenues à l'aide d'images appropriées. Ces illustrations permettent d'apprécier la distribution des habitats sur le site selon différents intervalles de valeurs de $I_{G}$ (ISH sur la figure 4), ainsi que leur sensibilité au débit (qualité, disponibilité, position). A titre d'exemple, les résultats relatifs aux habitats à tacons sont présentés à la figure 4 qui montre, à l'aide de l'indice global, que le milieu est dans l'ensemble très propice aux tacons. A faible débit, les meilleurs habitats $\left(I_{G}>0.7\right)$ occupent la portion centrale de l'écoulement. A débit moyen, la valeur générale du chenal central diminue mais l'ensemble du domaine d'écoulement est favorisé par l'accroissement des vitesses et des profondeurs qu'occasionne l'augmentation du débit. A fort débit, il se produit une baisse générale de la qualité et de la disponibilité des habitats, les meilleurs ayant alors tendance à se situer plus près des rives. Le site semble être moins favorable aux alevins, sans doute à cause de l'hydrodynamisme assez élevé de l'endroit (LECLERC et al., 1991a).

\subsubsection{Disponibilité des habitats en fonction du débit}

La figure 5 montre les aires pondérées utiles (APU\%) des habitats à alevins de même que les aires classées de chacune des catégories d'habitats pour l'ensemble du site à l'étude. Cette figure révèle que le site offre un niveau global d'aires pondérées utiles (APU\%) de $55 \%$ au débit optimum de $160 \mathrm{~m}^{3} / \mathrm{s}$; au-delà et en deçà de ce débit, le site perd rapidement de sa valeur. A ce débit, les habitats sont principalement constitués d'aires de moyenne $\left(0,4<I_{G}<0,7\right)$ et de très bonne qualité $\left(I_{G}>0,7\right)$. 

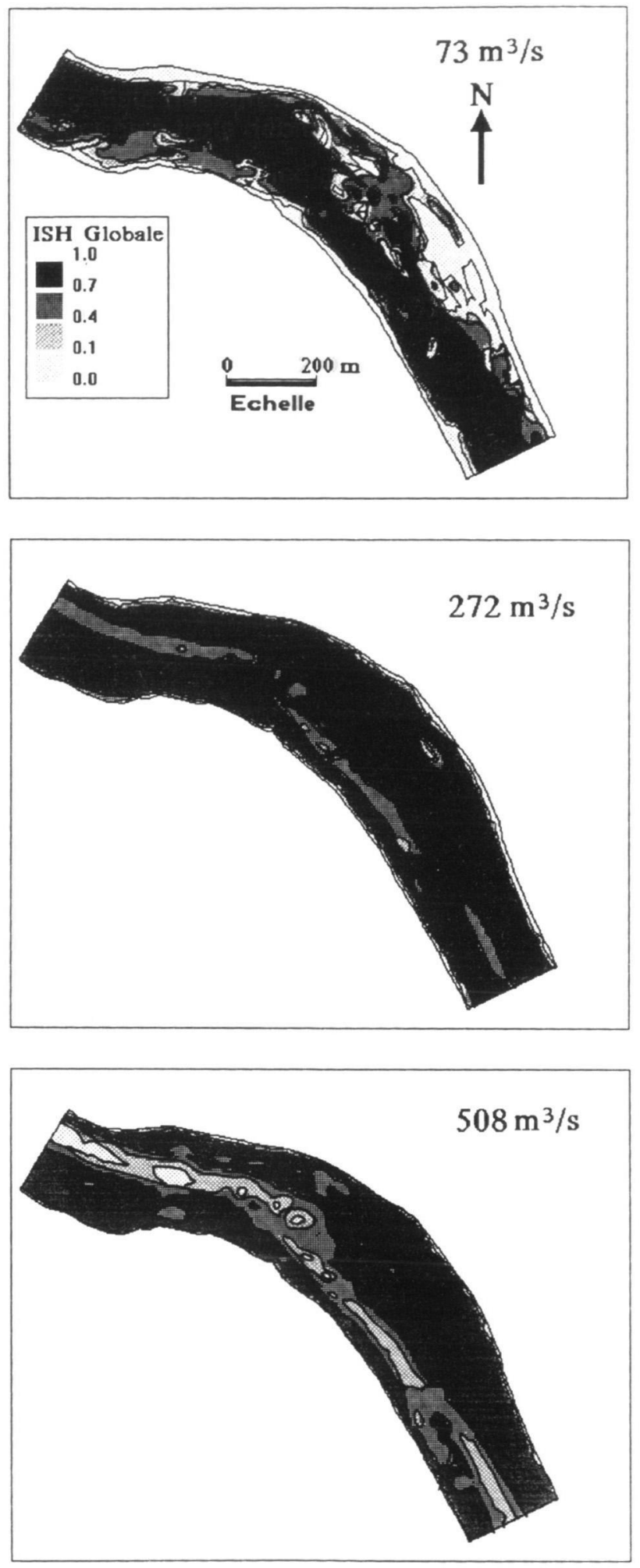

Figure 4 : Distribution spatiale des aires classées selon l'indice de sélectivité globale $\left(I_{G}\right)$ pour les tacons à trois débits différents.

Figure 4 : Mapping of the areas classified according to the global $H S I\left(I_{G}\right)$ for parr at three different discharges. 
Pour les tacons (figure 5), plus de $80 \%$ de la superficie du site dans le registre de débit $\left(140\right.$ à $300 \mathrm{~m}^{3} / \mathrm{s}$ ) est constituée d'habitats ayant une valeur optimale d'indice global $\left(I_{G}>0,7\right)$. Les habitats cotés 0,4 à 0,7 forment une bonne partie des autres aires disponibles. La valeur maximale des APU\% est atteinte pour un débit de $225 \mathrm{~m}^{3} / \mathrm{s}$. C'est avec une diminution de débit à partir de cette valeur que les habitats peuvent décroître rapidement en disponibilité. Une augmentation du débit produit également une diminution d'habitats à tacons, mais à un rythme moindre (figure 5).
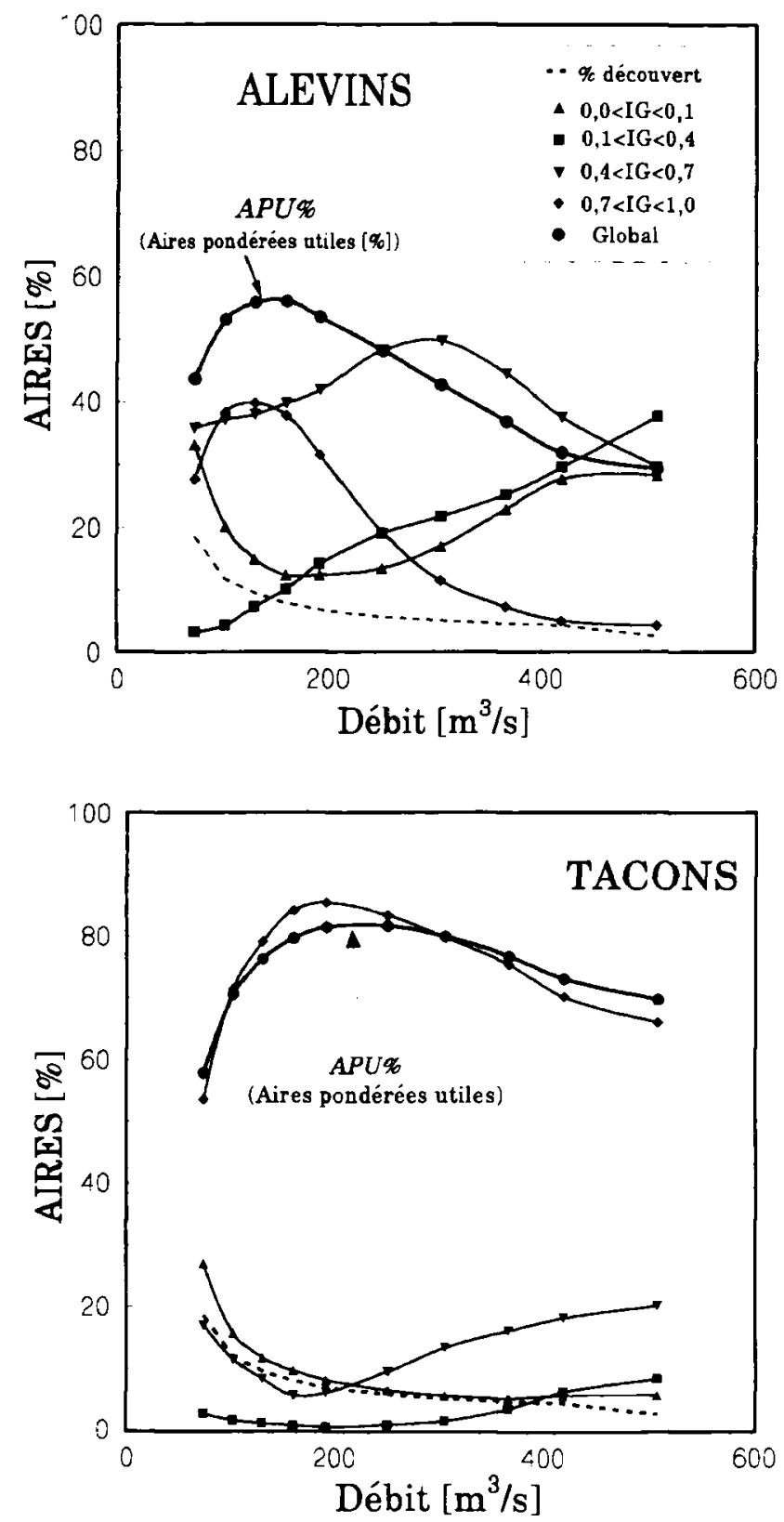

Figure 5 : Variation des aires pondérées utiles $(A P U \%)$ et des aires classées $\left(I_{G}\right)$ en fonction du débit pour les alevins et les tacons.

Figure 5 : Discharge sensitivity of Percentage Usable Areas (PUA) and different suitability intervals $\left(I_{G}\right)$ for fry and parr. 


\subsection{Mobilité des habitats}

\subsubsection{Coefficients de mobilité}

Les six zones homogènes définies dans le site sont représentées à la figure 6 . Dans chaque zone, les déplacements en fonction du débit des centroïdes des habitats de très bonne qualité $\left(I_{G}>0,7\right)$ sont représentés par des trajectoires. L'origine et la fin des trajectoires correspondent à la position moyenne des habitats aux débits de 73 et $508 \mathrm{~m} / \mathrm{s}$, respectivement. Le tracé des lignes représente les positions successives des centroïdes pour les différentes simulations. La longueur des lignes entre dans le calcul des coefficients de mobilité $(M)$, lesquels sont donnés aux tableaux III et IV.

Les zones 4 et 5 sont celles qui présentent les plus grands coefficients de mobilité pour les alevins $\left(M_{A}\right)$, ce qui s'explique par la morphologie de ces zones qui est relativement plate, étendue, et de faible pente. Les zones 2 et 6 présentent un plus faible coefficient de mobilité dû au fait que le thalweg tend à se rapprocher de la rive en ces endroits. La mobilité des habitats à tacons a un comportement similaire à celui des alevins, les plus forts coefficients de mobilité $\left(M_{T}\right)$ se retrouvant dans les zones de hauts-fonds plats comme ceux de la zone 5 (figure 6, tableau III).

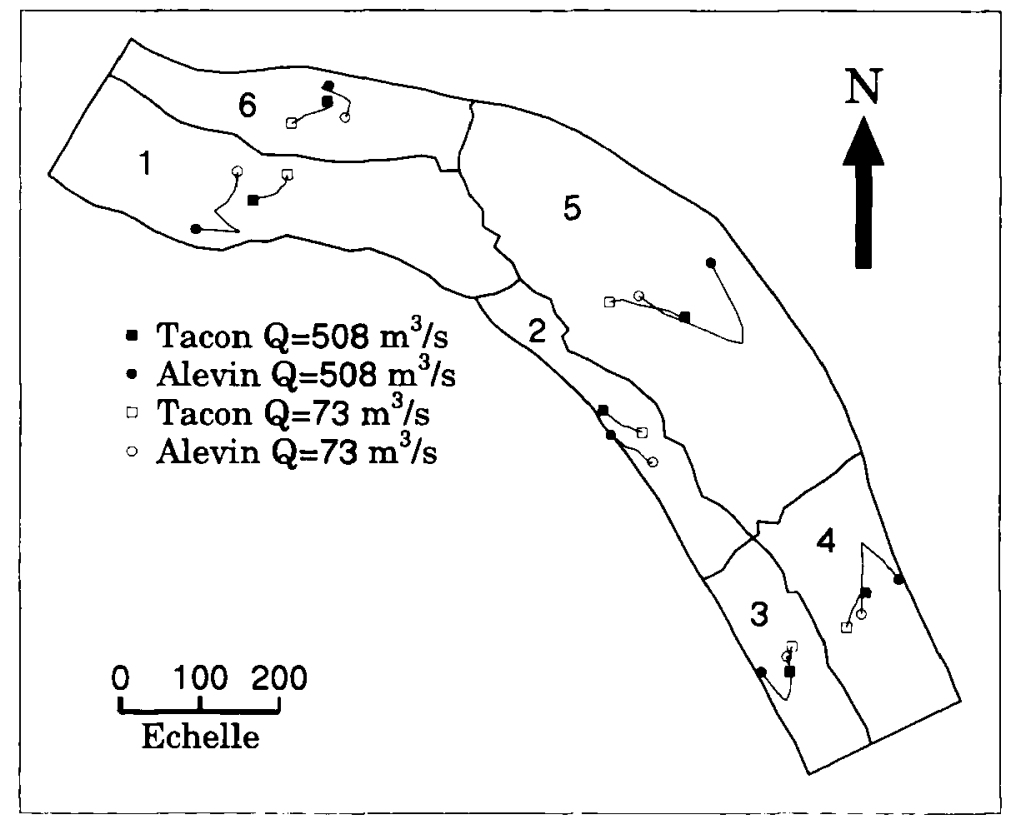

Figure 6 : Zones homogènes définies dans le site de modélisation et déplacement du centroïde des habitats de bonne qualité $\left(I_{G}>0,7\right)$ en fonction du débit pour les alevins et les tacons.

Figure 6 : Homogeneous zones defined within the modelled site and displacement of the centroid of the good quality habitats $\left(I_{G}>0,7\right)$ as a function of discharge for fry and parr.

\subsubsection{Vitesse de déplacement des habitats}

\subsubsection{Régime naturel de débit}

Pour les alevins, la vitesse de déplacement des habitats de bonne qualité $\left(I_{G}>0,7\right)$ en régime naturel est en moyenne de $0,18 \mathrm{~m} / \mathrm{h}$ (tableau III) et la vitesse de déplacement (de probabilité inférieure ou égale à $2 \%$ ) est de $0,32 \mathrm{~m} / \mathrm{h}$. La zone 5 , la plus riche en habitats à juvéniles, est aussi celle qui présente les vitesses de déplacement les plus élevées : $0,62 \mathrm{~m} / \mathrm{h}$ comme valeur de probabilité inférieure ou égale à $2 \%$. Ces données servent de référence pour l'analyse des conditions susceptibles de se produire avec l'hypothèse de régime d'exploitation. 
Tableau III : Vitesses de déplacement $D$ (mètres /heure) des centroïdes de l'habitat à alevins en régime naturel et d'exploitation. $M_{A}$ : coefficient de mobilité $\left(\mathrm{m} / \mathrm{m}^{3} / \mathrm{s}\right) ; \Delta Q / \Delta t$ : taux de variation du débit $\left(\mathrm{m}^{3} / \mathrm{s} / \mathrm{h}\right)$.

Table III : Velocity of displacement $D$ (metres per hour) for fry habitat centroids under natural and exploitation regimes. $M_{A}$ : coefficient of mobility $\left(\mathrm{m} / \mathrm{m}^{3} / \mathrm{s}\right) ; \Delta a / \Delta t$ : rate of discharge variation $\left(\mathrm{m}^{3} / \mathrm{s} / \mathrm{h}\right)$.

\begin{tabular}{lccccccccr}
\hline & & \multicolumn{4}{c}{ Régime naturel } & \multicolumn{3}{c}{ Hypothèse de régime d'exploitation } \\
\cline { 2 - 10 } & $M_{A}$ & Moyenne & $\delta Q_{50 \%}$ & $\delta Q_{10 \%}$ & $\delta Q_{2 \%}$ & Moyenne & $\delta Q_{50 \%}$ & $\delta Q_{10 \%}$ & $\delta Q_{2 \%}$ \\
\hline$\Delta \mathrm{Q} / \Delta t$ & - & 0,58 & 0,55 & 0,80 & 1,02 & 8,40 & 7,60 & 15,30 & 22,20 \\
Zone 1 & 0,37 & 0,22 & 0,21 & 0,30 & 0,38 & 3,10 & 2,80 & 5,70 & 8,20 \\
Zone 2 & 0,20 & 0,09 & 0,08 & 0,12 & 0,15 & 1,28 & 1,13 & 2,33 & 4,00 \\
Zone 3 & 0,28 & 0,13 & 0,13 & 0,18 & 0,23 & 1,97 & 1,72 & 3,53 & 6,16 \\
Zone 4 & 0,42 & 0,22 & 0,21 & 0,30 & 0,39 & 3,15 & 2,88 & 5,76 & 10,20 \\
Zone 5 & 0,61 & 0,36 & 0,34 & 0,49 & 0,62 & 5,10 & 4,60 & 9,30 & 16,40 \\
Zone 6 & 0,14 & 0,10 & 0,09 & 0,14 & 0,17 & 1,45 & 1,33 & 2,54 & 4,49 \\
Global & 0,34 & 0,18 & 0,17 & 0,25 & 0,32 & 2,64 & 2,37 & 4,73 & 8,27 \\
\hline
\end{tabular}

Concernant les habitats à tacons, on peut noter des comportements similaires à ceux observés pour les habitats à alevins (tableau IV). Cependant, les valeurs typiques sont inférieures d'un facteur voisin de 2. Ce comportement est attribuable à la plus grande disponibilité d'habitats à tacons sur le site, ce qui les rend moins susceptibles de mouvements importants associés à des variations de débit. Les changements de débit se traduisent plutôt par des variations graduelles de disponibilité.

\subsubsection{Hypothèse de régime d'exploitation}

On note au tableau III que la vitesse de déplacement des habitats de bonne qualité $\left(I_{G}>0,7\right)$ des alevins serait en moyenne de $2,6 \mathrm{~m} / \mathrm{h}$ en fonction du régime étudié, ce qui, par rapport au régime naturel, est près de 15 fois la moyenne et 8 fois les vitesses de déplacement de probabilité égale ou inférieure à $2 \%$. La zone 5 , qui est la plus riche en habitats à alevins, est aussi celle qui supporterait les vitesses de déplacement les plus élevées (tableau III).

Concernant les habitats à tacons, on note des comportements similaires à ceux observés pour les habitats à alevins, les valeurs typiques de vitesses de déplacement étant inférieures d'un facteur voisin de 2 (tableau IV). En moyenne, les vitesses de déplacement seraient de l'ordre de $1,2 \mathrm{~m} / \mathrm{h}$ (15 fois la moyenne et 8 fois les vitesses de probabilité égale ou inférieure à $2 \%$ en conditions naturelles). De nouveau, c'est la zone 5 , le plus riche habitat potentiel, qui présenterait les vitesses les plus élevées.

\section{DISCUSSION}

L'impact de fluctuations rapides et fréquentes du débit sur les habitats piscicoles peut être évalué d'au moins trois façons différentes (GORE et al., 1989) :

1) en considérant la valeur minimale d'Aires Pondérées Utiles (APU);

2) en déterminant les caractéristiques frontales de l'onde de lâcher ;

3) en évaluant la vitesse à laquelle les conditions d'habitat changent avec le débit.

Dans l'approche utilisée ici, l'emphase est mise sur le dernier aspect (dynamique), sans toutefois que le premier soit négligé. Ainsi, nous avons constaté que la disponibilité des APU est minimale aux débits extrêmes. Sous le régime d'exploitation considéré, les faibles débits seraient très fréquents pendant les périodes de stockage d'eau, et peuvent occasionner une dégradation importante de la qualité de l'habitat. En ce qui concerne la propagation du front de la crue, un relâchement graduel des eaux pourrait aider à minimiser ses effets négatifs. 
Tableau IV : Vitesse de déplacement $D$ (mètres/heure) des centroïdes de l'habitat à tacons en régime naturel et d'exploitation. $M_{A}$ : coefficient de mobilité $\left(\mathrm{m} / \mathrm{m}^{3} / \mathrm{s}\right) ; \Delta Q / \Delta t:$ taux de variation du débit $\left(\mathrm{m}^{3} / \mathrm{s} / \mathrm{h}\right)$.

Table IV : Velocity of displacement $D$ (metres per hour) for parr habitat centroids under natural and exploitation regimes. $M_{A}$ : coefficient of mobility $\left(\mathrm{m} / \mathrm{m}^{3} / \mathrm{s}\right) ; \Delta \mathrm{Q} / \Delta t:$ rate of discharge variation $\left(\mathrm{m}^{3} / \mathrm{s} / \mathrm{h}\right)$.

\begin{tabular}{lccccccccc}
\hline & \multirow{2}{*}{$M_{A}$} & \multicolumn{3}{c}{ Régime naturel } & \multicolumn{4}{c}{ Hypothèse de régime d'exploitation } \\
\cline { 3 - 10 } & & Moyenne & $\delta Q_{50 \%}$ & $\delta Q_{10 \%}$ & $\delta Q_{2 \%}$ & Moyenne & $\delta Q_{50 \%}$ & $\delta Q_{10 \%}$ & $\delta Q_{2 \%}$ \\
\hline$\Delta Q^{\prime} \Delta t$ & - & 0,58 & 0,55 & 0,80 & 1,02 & 8,4 & 7,6 & 15,3 & 22,2 \\
Zone 1 & 0,14 & 0,08 & 0,08 & 0,11 & 0,14 & 1,2 & 1,1 & 2,1 & 3,1 \\
Zone 2 & 0,14 & 0,08 & 0,08 & 0,11 & 0,14 & 1,2 & 1,1 & 2,1 & 3,1 \\
Zone 3 & 0,08 & 0,05 & 0,04 & 0,06 & 0,08 & 0,7 & 0,6 & 1,2 & 1,8 \\
Zone 4 & 0,12 & 0,07 & 0,07 & 0,10 & 0,12 & 1,0 & 0,9 & 1,8 & 2,7 \\
Zone 5 & 0,23 & 0,13 & 0,13 & 0,19 & 0,24 & 1,9 & 1,7 & 3,5 & 5,1 \\
Zone 6 & 0,14 & 0,08 & 0,08 & 0,11 & 0,14 & 1,2 & 1,1 & 2,1 & 3,1 \\
Global & 0,14 & 0,08 & 0,08 & 0,11 & 0,14 & 1,2 & 1,1 & 2,1 & 3,1 \\
\hline
\end{tabular}

Par rapport à la dynamique de l'habitat, les résultats sur la ouananiche montrent, qu'en moyenne, la mobilité des habitats les plus favorables pourrait augmenter d'un facteur d'environ 15 fois par rapport aux conditions naturelles. II est cependant très difficile d'établir a priori de quelle façon, et avec quelle intensité une telle variation pourrait affecter les populations naturelles parce qu'il n'existe presque pas d'informations disponibles à ce sujet concernant le saumon atlantique (CUSHMAN, 1985). On peut cependant avancer un certain nombre d'hypothèses. Les effets négatifs des opérations de pointe sur les ouananiches juvéniles peuvent être classifiés en cinq catégories principales :

1) diminution de la surface disponible pour l'établissement de territoires;

2) diminution de la disponibilité de nourriture (benthos et dérive d'invertébrés) (CUSHMAN, 1985 ; FISHER et LAVOY, 1972) ;

3) augmentation des dépenses énergétiques pour contrer les forts courants, chercher des abris et/ou établir des nouveaux territoires ;

4) diminution du temps alloué à la quête de nourriture ;

5) augmentation de la mortalité par échouage sur les berges asséchées et par l'entraînement vers l'aval dû aux conditions hydrodynamiques au front de l'onde de lâcher d'eau (CUSHMAN, 1985).

Les fluctuations fréquentes de débit pourront provoquer l'apparition d'une bande près des berges qui sera trop fréquemment asséchée pour permettre le maintien des poissons territoriaux (BRUSVEN et MACPHEE, 1976). Le modèle hydrodynamique prédit une réduction d'environ $20 \%$ de la superficie couverte à des débits faibles $\left(73 \mathrm{~m}^{3} / \mathrm{s}\right.$, figure 5$)$. Si de tels débits surviennent avec une fréquence trop élevée, des réductions nettes des aires d'habitat disponibles pour la ouananiche sont prévisibles, en particulier pour les alevins qui ont tendance à se placer près des berges.

La sensibilité des poissons aux variations extrêmes de débit dépend de l'espèce, du stade de développement et de leur activité au cours du cycle nycthéméral. Ainsi, des diminutions trop rapides du niveau d'eau peuvent faire échouer un grand nombre d'alevins du saumon atlantique (âge $0+$ ) sur le rivage, tandis que pour les tacons (âges $>1+$ ) l'effet de ces diminutions pourrait être moins important (HVIDSTEN, 1985). Plus près du thalweg, la rivière peut être soumise à de fortes et fréquentes fluctuations de vitesse et de profondeur. La réponse possible des jeunes saumons à ces fluctuations pourrait être, d'une part, de se réfugier dans le substrat pendant les périodes de fort débit, sacrifiant ainsi une 
partie du temps alloué à la quête de nourriture. Ce type de réponse semble être celle du chabot nord-américain Cottus carolinae Gill, soumis à un régime d'exploitation de pointe (GORE, 1989). Également, HEGGENES (1988) a trouvé que les truites brunes Salmo trutta L. de taille égale ou supérieure à $67 \mathrm{~mm}$ n'étaient pas entraînées par le courant lors d'augmentations rapides de débit dans une rivière de la Norvège. Cet auteur conclut que la présence de substrats grossiers (e.g., galets et blocs) permet aux truites de trouver refuge au fond lors de forts débits. D'autre part, il a été bien démontré chez d'autres espèces de salmonidés que les individus ont tendance à occuper les sites qui fournissent un gain net maximal d'énergie (HUGHES et DILL, 1990). En ce sens, les indices de sélectivité reflètent dans une certaine mesure les positions de ces sites optimaux. Étant donné que la position de ces sites dans la rivière dépend en partie de la vitesse et de la configuration du fond, ils changent nécessairement avec le débit. Ainsi, les jeunes saumons pourraient être forcés soit de se déplacer en permanence à des meilleurs endroits, soit d'établir leur territoire dans des sites possédant des conditions optimales mais pour une durée écourtée pendant la période de croissance (HEGGENES, 1988). Finalement, concernant le cycle nycthéméral, des observations sur d'autres espèces de salmonidés de l'Amérique du Nord démontrent que les poissons se déplacent vers les berges pendant la nuit (EDMUNSON et al., 1968). Si un tel comportement est aussi le cas chez les saumons atlantiques juvéniles, il y aurait un risque de mortalité plus grand pendant la nuit que le jour.

Étant donné l'absence d'informations, au niveau biologique, il est difficile d'établir le degré de variation qui pourrait être toléré par l'espèce sans occasionner des diminutions importantes de densité. Une façon d'aborder le problème serait de recourir aux statistiques hydrologiques naturelles. On sait que des événements hydrologiques sévères peuvent arriver avec une certaine récurrence dans les rivières à saumons en conditions naturelles. Les populations de jeunes saumons ont néanmoins été capables de résister à de telles variations au cours des années, ce qui a permis la survie de l'espèce dans les cours d'eau. On peut utiliser ces événements extrêmes comme cadre de référence pour envisager divers scénarios d'exploitation. Dans la présente étude, la valeur de la ressource en cause oblige à considérer le pire cas possible, illustré par un régime d'exploitation de pointe d'une centrale produisant de l'énergie à sa capacité maximale. Dans un tel cas, les taux de mobilité des habitats dépasseraient largement ceux survenant avec une probabilité inférieure à $2 \%$. Ce déplacement des habitats pourrait être néfaste aux jeunes ouananiches en augmentant le taux de mortalité et en diminuant leur croissance, en raison de la diminution de la nourriture benthique et de l'augmentation des dépenses énergétiques reliées à l'adaptation à ce régime imposé. La production salmonicole (nombre de saumoneaux en dévalaison) de la rivière pourrait en conséquence subir une baisse. Ces impacts appréhendés à l'aide de la modélisation d'habitat ont permis d'écarter rapidement l'hypothèse de régime d'exploitation de pointe sur l'Ashuapmushuan.

Par rapport à la modélisation hydrodynamique, au moins deux sources possibles d'erreur sont à considérer. D'une part, nous avons effectué les simulations en supposant un état quasi permanent (peu de variation de la pente de la ligne d'eau dans le temps). Étant donné que l'ouvrage hydroélectrique impose des états fortement transitoires, une certaine erreur de calcul est probable. Cependant, le site modélisé $(1,4 \mathrm{~km})$ est suffisamment long pour amortir cette variation. Un calcul approximatif suggère qu'une telle variation devrait être dans l'ordre de grandeur de l'erreur de calcul de la méthode. D'autre part, l'onde de lâcher d'eau a tendance à diminuer en intensité (la pointe) et à augmenter en durée en se déplaçant vers l'aval (le phénomène du laminage). A nouveau, nous avons choisi une approche conservatrice en modélisant le pire cas possible, comme si le site était placé immédiatement en aval du barrage (de fait, il se situe à environ $3 \mathrm{~km}$ en aval d'un des ouvrages projetés). En pratique, il est probable que la mobilité de l'habitat diminuerait progressivement vers aval, amortie par le phénomène de laminage de l'onde. Cet énoncé est un corollaire des observations de GORE et al. (1989) concernant la diminution progressive vers l'aval de la variabilité temporelle des habitats (les APU) associée à la propagation d'une onde de lâcher d'eau.

Nous avons calculé la mobilité des habitats de chaque zone en fonction d'un "centroïde" à l'intérieur d'une zone. Celui-ci ne représente pas la position d'un poisson moyen qui se déplace avec le débit, mais plutôt une façon de décrire le déplacement de l'habitat. Cependant, les poissons territoriaux subissent un changement de conditions de 
I'habitat proportionnel aux déplacements du centroïde. Une approche qui considérerait les taux de variations de la qualité de l'habitat dans le temps à des points fixes de la rivière (des territoires potentiels) pourrait être plus utile pour les animaux moins mobiles et pour les frayères.

\section{CONCLUSION}

Une méthode d'estimation de la dynamique de l'habitat est proposée en vue d'une utilisation éventuelle pour la conception de régimes d'exploitation hydroélectriques adaptés à la conservation des espèces lotiques. Les résultats de son application dans la rivière Ashuapmushuan ont permis d'éliminer rapidement une hypothèse préliminaire d'exploitation classique en régime de pointe en raison de la forte variation spatio-temporelle qui aurait été imposée à l'habitat des ouananiches juvéniles. La méthode est une première approximation du problème et elle doit être perfectionnée, particulièrement pour améliorer la signification biologique qu'on lui prête. Dans ce sens, une augmentation des connaissances concernant la réponse des communautés aquatiques à divers régimes d'exploitation est nécessaire.

\section{REMERCIEMENTS}

Nous tenons à remercier Messieurs André BOUDREAULT du Groupe Environnement Shooner Inc. et Louis BERNATCHEZ, professeurs à I'INRS-Eau, pour leurs nombreuses suggestions lors de la rédaction du manuscrit. Nous remercions également la viceprésidence Environnement d'Hydro-Québec, pour son support financier à cette étude et pour l'autorisation de publier cet article.

\section{BIBLIOGRAPHIE}

BOBÉE B., ROBITAILLE R., 1977. The use of the Pearson type 3 and log-Pearson type 3 distributions revisited. Wat. Res. Research, 13, 427-443.

BOUDREAULT A., LECLERC M., BELLEMARE J.F., SHOONER G., 1989. Étude des répercussions du détournement de la rivière Aux Pékans sur les habitats salmonicoles de la rivière Moisie. Rapport de Gilles Shooner et Associés et de l'INRS-Eau à la Direction Environnement d'Hydro-Québec, Québec, 120 p., 3 annexes.

BOVEE K.D., 1978. The incremental method of assessing habitat potential for coolwater species, with management implications. Am. Fish. Soc. Spec. Pub., 11, 340-346.

BRUSVEN M.A., MACPHEE C., 1976. The effect of river fluctuations resulting from hydroelectric peaking on selected aquatic invertebrates and fish. Res. Tech. Compl. Repet. Proj. A. 36. IDA 40, 46 p.

COTÉ Y., DULUDE P., JOMPHE D., LABELLE J.P., ROULEAU A., ROY L., 1987. Essai de classification normalisée des substrats granulaires et des faciès d'écoulement pour l'évaluation de la production salmonicole. Ministère du Loisir, de la Chasse et de la Pêche, Direction générale de la Faune, Québec, $10 \mathrm{p}$.

CUSHMAN R.M., 1985. Review of ecological effects of rapidly varying flows downstream from hydroelectric facilities. N. Am. J. Fish. Man., 5, 330-339.

DHATT G., TOUZOT G., 1981. Une présentation de la méthode des éléments finis. Presses de l'Université Laval, Québec, et Maloine, Paris, 543 p.

EDMUNSON E., EVEREST F.E., CHAPMAN E.D., 1968. Permanence of station in juvenile Chinook salmon and Steelhead trout. J.Fish.Res.Bd.Can., 25, 1453-1464.

FISHER S.G., LAVOY A., 1972. Differences in littoral fauna due to fluctuating water levels below a hydroelectric dam. J. Fish. Res. Bd. Can., 29, 1472-1476.

GARCIA DE JALON D., MONTES C., BARCELO E., CASADO C., MENES F., 1988. Effects of hydroelectric scheme on fluvial ecosystems within the Spanish Pyrenees. Regulated Rivers, 2, 479-491.

GISLASON J.C., 1985. Aquatic insect abundance in a regulated stream under fluctuating and stable diel flow patterns. N. Am. J. Fish. Man., 5, 39-46. 
GORDON N.D., McMAHON T., FINLAYSON B.L., 1992. Stream hydrology. An introduction for ecologists. John Wiley \& Sons, Chichester, $526 \mathrm{p}$.

GORE J.A., 1989. Case histories of instream flow analyses for permitting an environmental impact assessment in the United States. Sth. Afr. J. Aquat. Sci., 16, 194-208.

GORE J.A., NESTLER J.M., LAYZER J.B., 1989. Instream flow predictions and management options for biota affected by peaking-power hydroelectric operations. Regulated Rivers, 3, 35-48.

HAVEY K.A., WARNER K., 1970. The landlocked Salmon (Salmo salar). Its life history and management in Maine. Sport Fish. Inst., Washington and Maine Dept. Inland Fish. Game, $129 \mathrm{p}$.

HEGGENES J., 1988. Effects of short-term fluctuations on displacement of, and habitat use by, brown trout in a small stream. Trans. Am. Fish. Soc., 117, 336-344.

HUGHES N.F., DILL L.M., 1990. Position choice by drift-feeding salmonids : model and test for Arctic Grayling (Thymallus arcticus) in subarctic mountain streams, interior Alaska. Can. J. Fish. Aquat. Sci., 47, 2039-2048.

HVIDSTEN N.A., 1985. Mortality of pre-smolt Atlantic salmon, Salmo salar L., and brown trout, Salmo trutta L., caused by fluctuating water levels in the regulated river Nidelva, central Norway. J. Fish Biol., 27, 711-718.

LECLERC M., DHATT G., ROBERT G.L., TESSIER J.C., SOULAIMANI A., DUPUIS P., MATTE Y., 1987. Modélisation des écoulements de l'Archipel de Montréal par éléments finis : aspects divers de l'application. Rev. Int. Sci. Eau, 3, 41-56.

LECLERC M., BELLEMARE J.F., DUMAS G., DHATT G., 1990. A finite element model of estuarian and river flows with moving boundaries. Adv. Wat. Res., 4, 158-168.

LECLERC M., BOUDREAU P., BELZILE L., 1991a. Aménagement hydroélectrique de l'Ashuapmushuan. Avant-projet, phase 1. Étude environnementale de la faune ichtyenne. Volume 3 : Modélisation numérique des habitats à ouananiche d'un tronçon représentatif de la rivière Ashuapmushuan ( $\mathrm{km} 68$ ). Rapport de l'Institut National de la Recherche Scientifique-Eau No R-316 et du Groupe Environnement Shooner Inc., pour la Vice-Présidence Environnement, Hydro-Québec, Montréal, 76 p., 4 annexes.

LECLERC M., BOBÉE B., BOUDREAULT A., SHOONER G., CORFA G., 1991b. Instream Flow Incremental Methodology and 2-D hydrodynamic modeling: efficient tools to determine guaranteed minimum flow for biological purposes, in Ouazar D., Ben Sari D., Brebbia C.A., Computer methods in water resources II. Vol 2 : computational hydraulics and hydrology, 289-300, Computational Mechanics Publications, Southampton and Springer-Verlag, Berlin.

MATHUR D., BASON W. H., PURDY E. D. Jr., SILVER C.A., 1985. A critique of the instream flow incremental methodology. Can. J. Fish. Aquat. Sci., 42, 825-831.

MINSHALL G.W., 1985. Aquatic insect-substratum relationships in Resh V.H. et Rosenberg D.M., The ecology of aquatic insects, 358-399, Praeger, New York.

NESTLER J.M., MILHOUS R.T., LAYZER J.B., 1989. Instream habitat modeling techniques in Gore J.A., Petts G.E., eds., Alternatives in regulated river management, 295-315, CRC Press Inc., Boca Raton, Florida.

REISER D.W., WESCHE T.A., ESTES C., 1989. Status of instream flow legislation and practices in North America. Fisheries, 14, 22-29.

RIMMER D.M., 1980. On the automnal habitat change of juvenile Atlantic Salmon (Salmo salar). Thèse de Doctorat, Université du Nouveau-Brunswick, Canada, $192 \mathrm{p}$.

SCOTT D., SHIRVELL C.S., 1987. A critique of the instream flow incremental methodology and observations on flow determination in New Zealand, in Craig J.F., Kemper J.B., eds., Regulated Streams. Advances in Ecology, 27-43, Plenum Press, New York. 
SOUCHON Y., TROCHERIE F., FRAGNOUD E., LACOMBE C., 1989. Les modèles numériques des microhabitats des poissons : application et nouveaux développements Rev. Sci. Eau, 2, 807-830.

SYMONS P.E., HELAND M., 1978. Stream habitats and behavioral interactions of underyearling and yearling atlantic salmon (Salmo salar). J. Fish. Res. B. Can., 35, 175183.

VALENTINE M., 1991. Aménagement hydroélectrique de l'Ashuapmushuan. Avant-projet, phase 1. Etude environnementale de la faune ichtyenne. Volume 1 : Synthèse des connaissances sur la ouananiche et les autres espèces ichtyennes. Centre écologique du lac Saint-Jean inc. et Groupe Environnement Shooner Inc., pour la Vice-Présidence Environnement, Hydro-Québec, Montréal, $76 \mathrm{p}$. 